J. Product. \& Dev., 17(2): 223 - 228(2012)

\title{
STRUCTURE DISTRIBUTION AND DENSITY OF THE MUSCLE SPINDLES IN GASTROCNEMIUS MUSCLE OF Melopsittacus undulates
}

\section{A. El-Tarhonui and M. Ali El-Zian}

Department of Zoology, Faculty of Science, Benghazi University, Libyia.

\begin{abstract}
Five adult Melopsittacus undulates birds were used to study the morphology, density and distribution of the muscle spindles in gastrocnemius muscles in both right and left legs, employing serial transverse histologic sections stained with hematoxylin and eosin. The density of such muscle spindles was 100.95/gm. They were scattered within the whole muscle. Each spindle showed four to less intrafusal muscle fibers and blood capillaries and enclosed in outer capsule. A complex spindle was seen in paired arrangement in the belly of the muscle.
\end{abstract}

Key words: Muscle spindles, gastrocnemius muscle, Melopsittacus undulates.

\section{INTRODUCTION}

A muscle spindle in vertebrate skeletal muscles consists of specialized muscle fibers enclosed by fusiform capsule. These specialized intrafusal muscle fibers are smaller in size than the surrounding extrafusal muscle fibers that constitute the whole muscle. It has been shown by light microscopy that two different types of intrafusal muscle fiber occur in the muscle spindles of cat (Boyd 1960 and 1962) a type in which the nuclear bag fiber is long and thick with a characteristic bag of nuclei aggregating at the mid-equatorial region of spindle and a type in which the nuclear chain fiber is short and thin with nuclei arranged in a row at the central region.

These intrafusal muscle fiber types have also been shown to occur in spindles of different mammalian species (Barker and Banks 2004).

Therefore, the aim of this study structure distribution and density of the muscle spindles in gastrocnemius muscle of melopsittacus undulates, in Libia. 


\section{MATERIALS AND METHODS}

Five adult Melopsittacus undulates birds of both sexes and with an average weight of $30 \mathrm{mg}$ were used in this experiment. The birds were killed by using chloroform. Gastrocnemius muscle of both left and right legs were dissected from their attached tendons. Specimens from the muscles were collected and subjected to the histological technique and staining (Bancroft and Gamble 2008). They were firstly fixed in $10 \%$ buffered neutral formalin solution to preserve the cell-structure for 24 hrs. these specimens were prepared to be cut thin sections for examination on a light microscope. This process required the specimens were embedded in supported medium that can properly mounted sectioned with a rotary-mcrotome. Such embedding required that the specimens were firstly dehydrated in ascending grades of ethanol (50100\%). The dehydrated specimens cleared of the alcohol and tissue (become transparent) by incubating in xylene. Once specimens were properly dehydrated and cleared, they were embedded in paraffin wax. Blocking and sectioning (serial sections of 5-micron thick) were prepared then mounted on clean slides. The latter left to dry in an oven at $37^{\circ} \mathrm{C}$ for $12 \mathrm{hrs}$. Later on, these sections were stained by hematoxylin and eosin stain (H\&E) and mounted in DPX.

\section{RESULTS}

All spindles were situated in parallel with the extrafusal fiber bundles. They occurred as a single structure, although there were some complex spindles. These receptors were readily distinguished in serial transverse sections by their prominent capsule. Each spindle was surrounded by a capsule consisted of concentric layers of connective tissue, the thickness of the capsule was greater in the equatorial region than the polar region. Based on the pattern of equatorial nucleation three types of intrafusal fibers were identified, $\mathrm{bag}_{1}, \mathrm{bag}_{2}$ and chain fibers (Figure 1). In equatorial regions the intrafusal muscle fibers contained one nucleus, they were darkly stained lying somewhat eccentrically within the fiber. At juxtaequatorial region, the nuclei lie quite close to each other while in the polar region the nuclei were located deeper within the fibers. Blood capillaries were observed associated with the capsule (Figure 2). A few complex spindles were found in the form of paired associated in which two or three spindles lay side by side (Figures 3 and 4). 


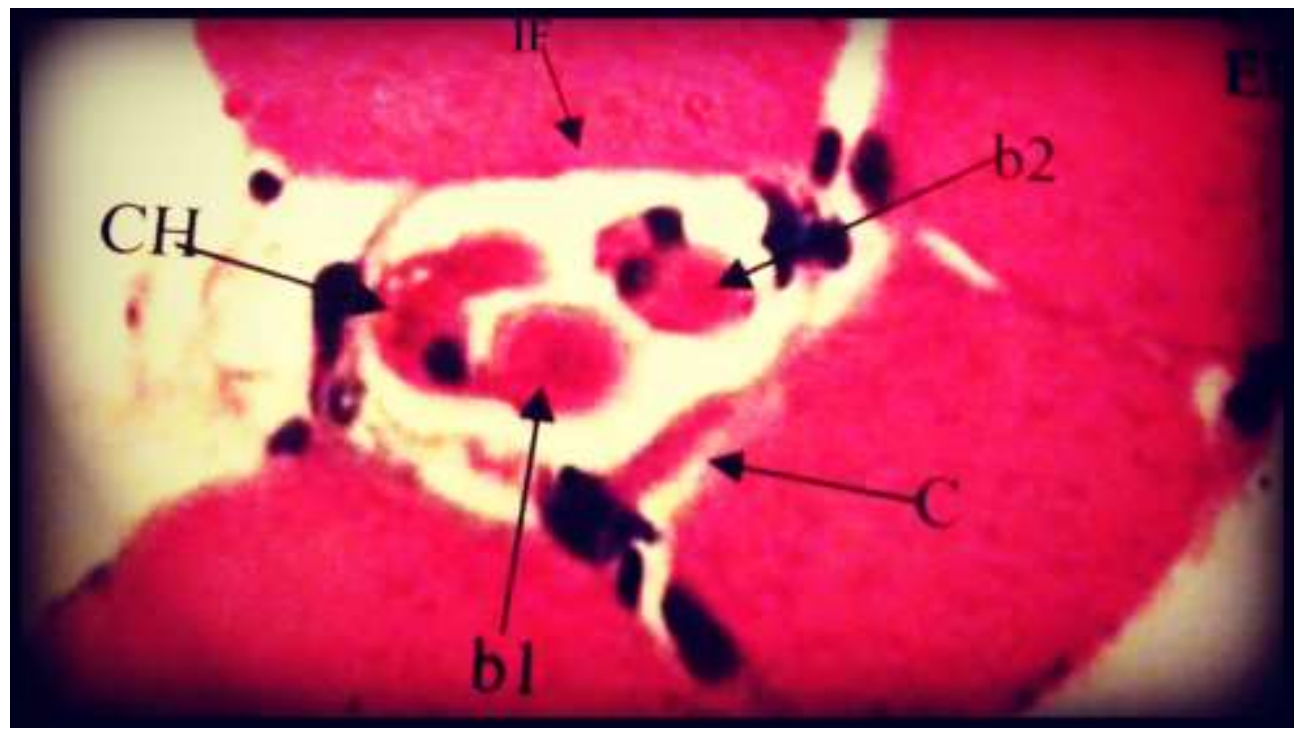

Figure (1): A photomicrograph of transverse section showing muscle spindle and the surrounding extrafusal muscle fibers at the equatorial region $\mathrm{EF}$. Extrafusal muscle fiber, IF. Intrafusal muscle fiber, $\mathrm{b}_{1}$. \& $\mathrm{b}_{2} . \mathrm{Bag}_{1} \&$ bag $_{2}$, NC. Nuclear chain fibers, C. Capsule, H\&E x 600.

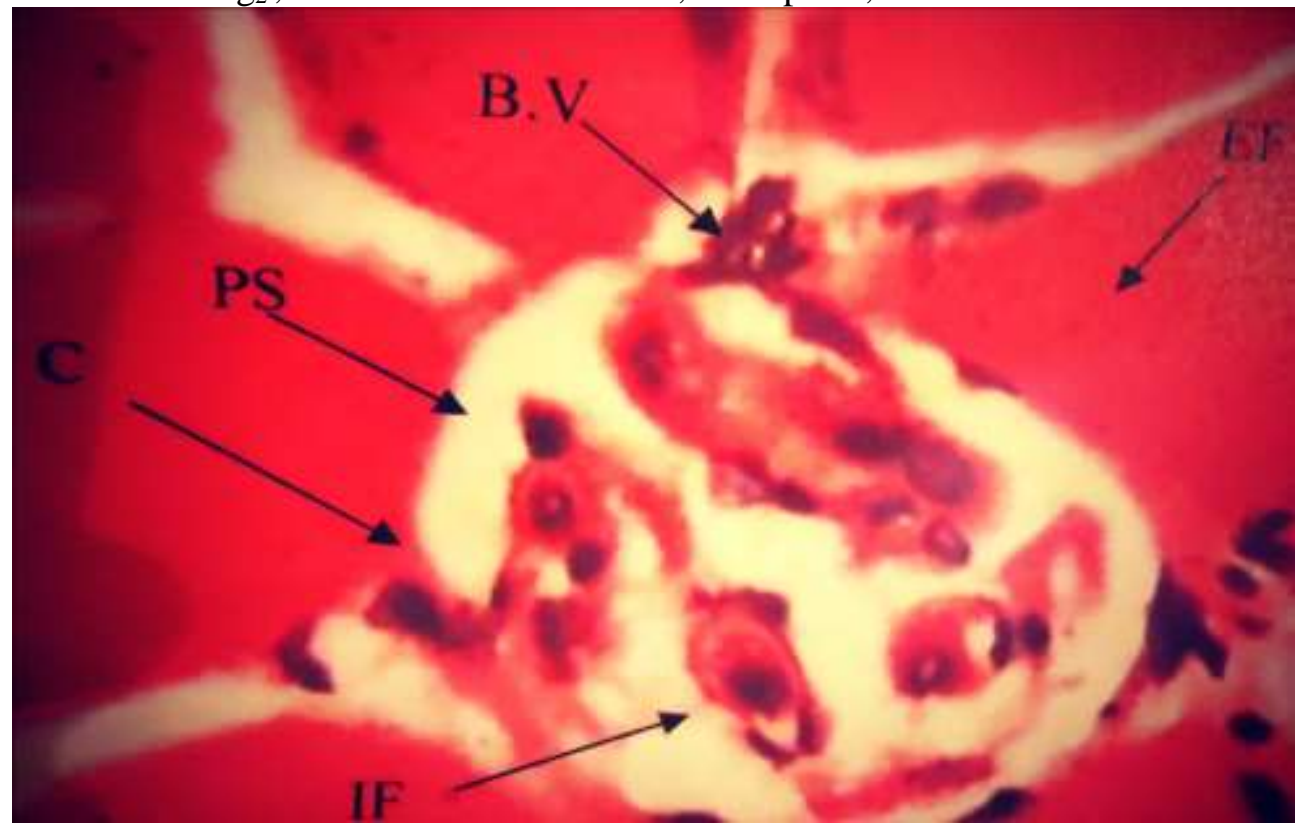

Figure (2): A photomicrograph of transverse sections through the equatorial region showing blood vessel associated with the capsule of the muscle spindle IF- intrafusal muscle fibers, EF- extrafusal muscle fibers, BV- blood vessel, PS- periaxial space and C- capsule, H \& E x 600. 


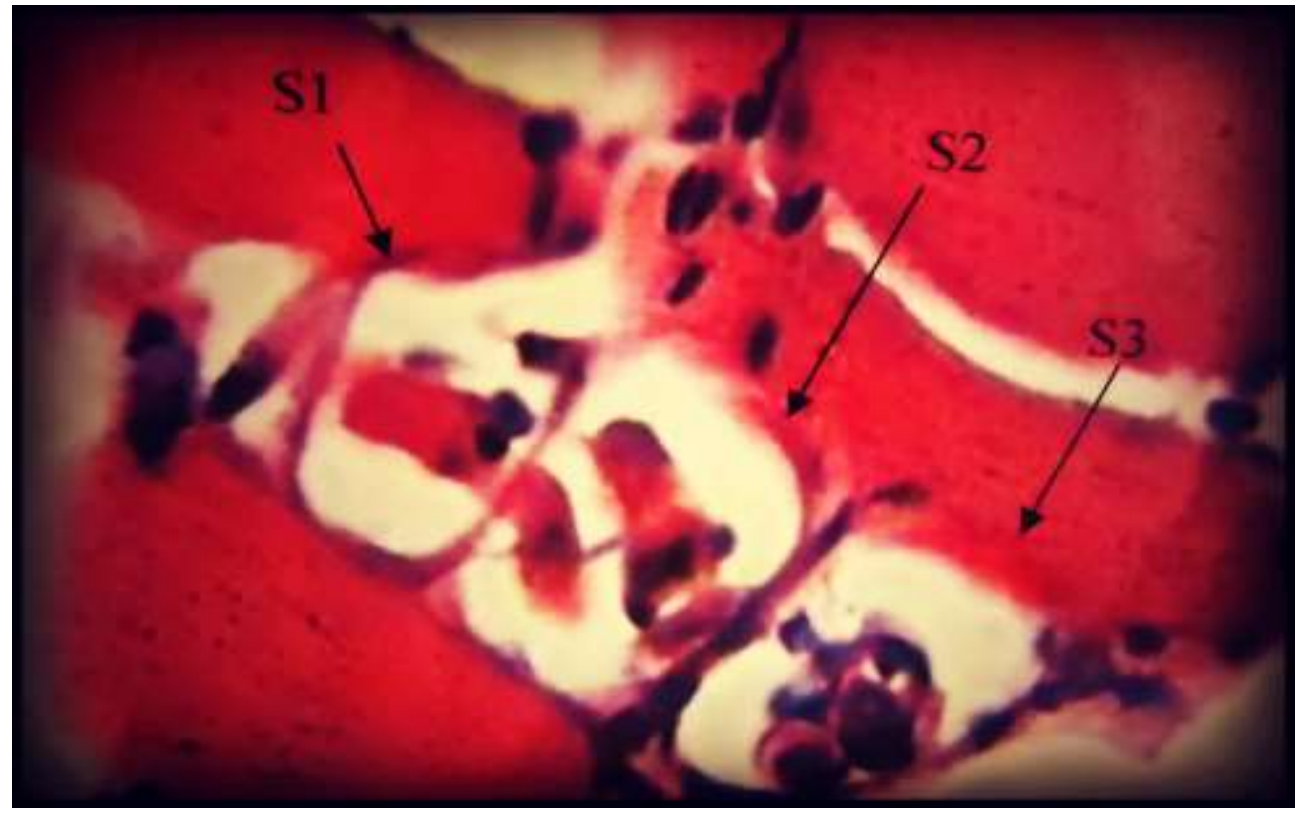

Figure (3): A Photomicrograph showing a paired linkage, three muscle spindles lay side by side to each other. $S_{1}, S_{2}$ and $S_{3}, H \& E$ x 600 .

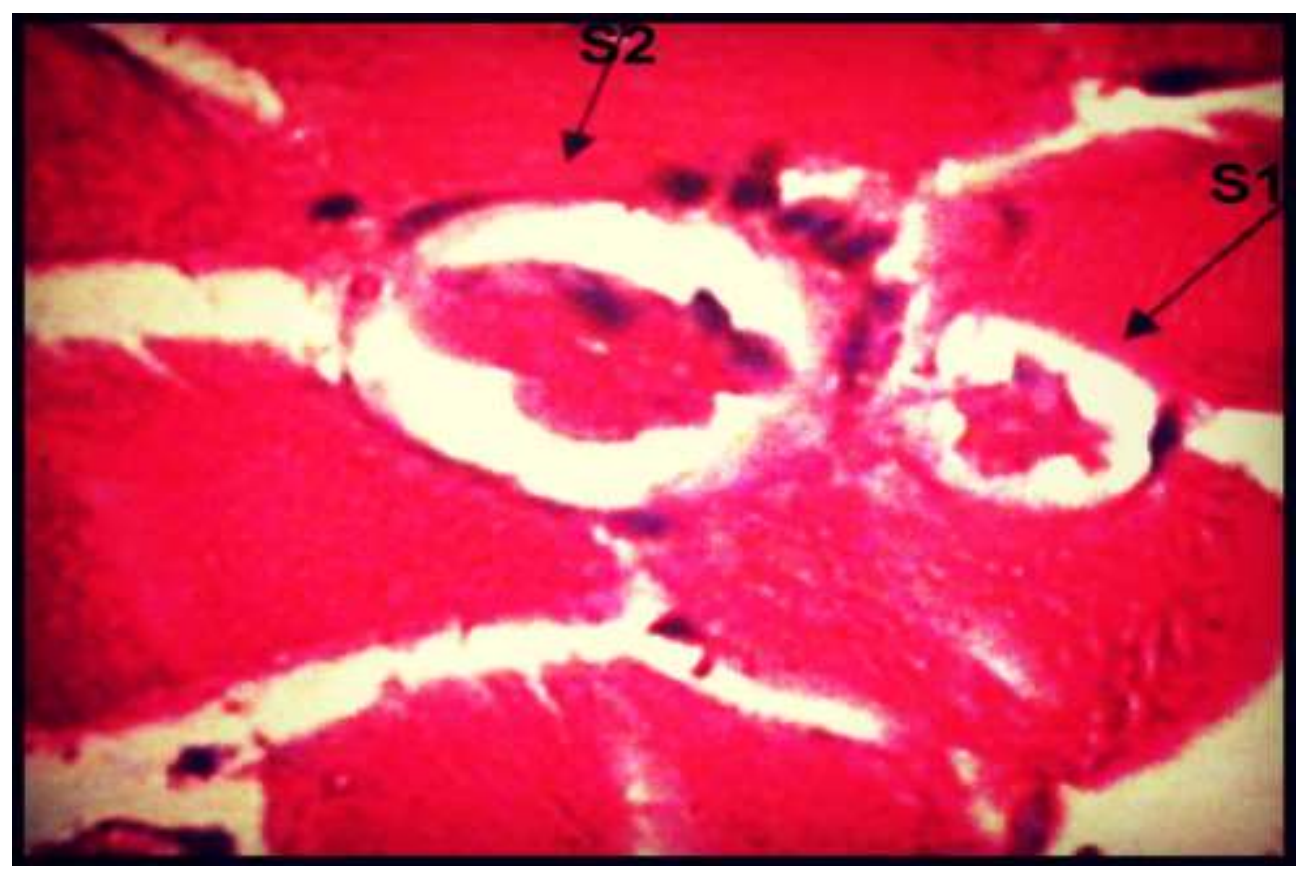

Figure (4): Photomicrograph showing complex spindle in which one at equatorial region and the other at juxtaequatorial region. $S_{1}$ and $S 2, H \& E \times 600$. 


\section{DISCUSSION}

In the present study, the structure of muscle spindles of gastrocnemius muscle in Melopsittacus undulates was found to be similar to that observed in other species (Cooper 1960). It consists of capsules, lymphatic space, intrafusal muscle fibers in varying numbers. This study also indicates that the spindles in the Melopsittacus undulates birds are highly vascularized, the blood capillaries entered the periaxial space from the equatorial region, similar finding has been described in the rabbit spindles (El-tarhouni and Almoughabi, 2006). The presence of many intrafusal capillaries may be related to the more oxygen and metabolic demands of the intrafusal muscle fibers. The spindles occur singly and few were combined in pairs in which the intrafusal bundles either remain separated encapsulated (inner capsule) or shared a common capsule (outer capsule). The same observation has been shown by Bakker and Richmond (1981) in cat neck and intervertebral muscles in which many units are linked together in tandem complex. The majority of scattered spindles that found along the whole length of the gastrocnemius muscle in Melopsittacus undulates were concentrated in the belly of the muscle near the region of nerve entry. These results are in accordance with that of Yellin (1969). The present study also revealed that the mean density of muscle spindles in gastrocnemius muscle was $100.95 \mathrm{gm}$. The previous results reflect the pattern of life and locomotion (El-Tarhouni, 2010).

\section{REFERENCES}

Bancroft, J. D. and Gamble, M. (2008). Theory And Practice Histological Techniques. $5^{\text {th }}$ ed., Chuchill Livingston and Edinburgh, London and New York.

Barker, D. and Banks, R.W. (2004). The spindle. In: Myology (ed. A.G. Engel and B. Q. Barker). $3^{\text {rd }}$ ed, McGraw-Hill, New York, pp. 333660.

Bakker, G.J. and Richmond, F.J. (1981). Two types of muscle spindles in cat-neck muscles: a histochemical study of intrafusal fiber compostion. Journal of Neurophysiology, 45: 973-986.

Boyd, I.A. (1960). The diameter and distribution of the nuclear bag and nuclear chain muscle fibers in the muscle spindles of the cat. Journal of Physiology, (Lond), 153: 23-24. 
Boyd, I . A. (1962). The structure innervations of nuclear bag muscle fiber system and nuclear chain muscle fiber system in mammalian muscle spindles. Phil. Tran R. Soc. B; 245: 81-136.

Cooper, S. (1960). Muscle spindles and other muscle receptors. In Bourne G. H.(ed). The Structure And Function Of Muscle. New York; Academic Press, 381-420.

El-Tarhouni. A. (2010). Comparison in the structure of lizards muscle spindles. Zagazig Veterinary Journal, 38 (1): 67-88.

El-Tarhouni, A. and Almoughrabi., F. (2006). The structure and distribution of muscle spindles in Extensor carpio radialis longus of rabbit. Journal of Egyptain Ger. Soc. Zool., 51c: 57-64.

Yellin, H. (1969). A histological study of muscle spindles and their relationship to extrafusal fiber types in the rat. Journal of Anat., 125: 31 .

\section{كثافة وتوزيع وتركيب المغآزل العضلية في عضلة بطن الساق لطائر

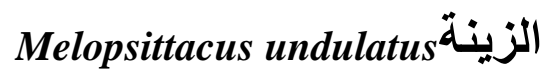

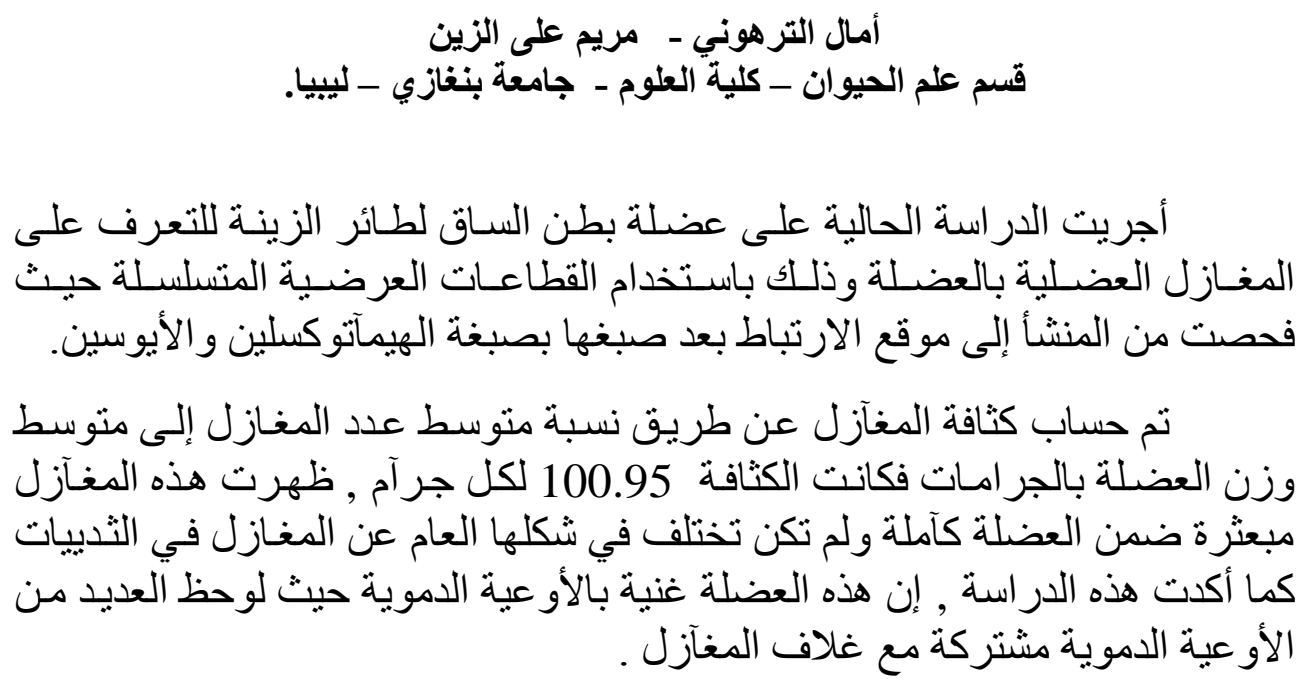

